Bulletin of the Section of Logic

Volume 48/3 (2019), pp. 207-211

http://dx.doi.org/10.18778/0138-0680.48.3.04

Marcin Łazarz

\title{
A NOTE ON DISTRIBUTIVE TRIPLES
}

\begin{abstract}
Even if a lattice $L$ is not distributive, it is still possible that for particular elements $x, y, z \in L$ it holds $(x \vee y) \wedge z=(x \wedge z) \vee(y \wedge z)$. If this is the case, we say that the triple $(x, y, z)$ is distributive. In this note we provide some sufficient conditions for the distributivity of a given triple.

Keywords: Distributive triple, dually distributive triple, covering diamond.

Standard lattice-theoretic notions can be found in [3]. Let us recall basic definitions and facts. If $L$ is a lattice and $a, b \in L$, then the set $[a, b]=\{c \in L: a \leqslant c \leqslant b\}$ is called an interval (in $L$ ). Clearly, any interval is a sublattice of $L$. If $X \subseteq L$, then $[X]$ stands for the sublattice generated by $X$, i.e., the smallest sublattice of $L$, which contains the subset $X$. For any subset $X \subseteq L$ and for any interval $[a, b]$ we define

$$
\llbracket a, b \rrbracket_{X}:=[a, b] \cap[X] .
$$

In particular, if $X=\{x, y, z\}$, then $\llbracket x \wedge y \wedge z, x \vee y \vee z \rrbracket_{X}=[X]$.

A lattice $L$ is said to be modular if $x \leqslant z$ implies $(x \vee y) \wedge z=x \vee(y \wedge z)$, for all $x, y, z \in L$. Moreover, $L$ is called distributive if $(x \vee y) \wedge z=$ $(x \wedge z) \vee(y \wedge z)$, for all $x, y, z \in L$. The Dedekind-Birkhoff Theorem (cf. [3], p. 59) states that a lattice $L$ is modular if and only if $L$ does not contain a sublattice isomorphic to $N_{5}$ (so-called pentagon), and moreover, and $L$ is distributive if and only if $L$ does not contain a sublattice isomorphic to $N_{5}$ nor $M_{3}$ (so-called diamond).
\end{abstract}


Let $L$ be an arbitrary lattice and $x, y, z \in L$. We say that $(x, y, z)$ is a distributive triple, $(x, y, z) D$ in symbols, if $(x \vee y) \wedge z=(x \wedge z) \vee(y \wedge z)$. Similarly, $(x, y, z)$ is called a dually distributive triple, $(x, y, z) D^{*}$ in symbols, if $(x \wedge y) \vee z=(x \vee z) \wedge(y \vee z)$ (cf. [7], p. $\left.76^{1}\right)$. Clearly, $L$ is distributive if and only if $(x, y, z) D$, for all $x, y, z$. G. Birkhoff proved the following.

Theorem 1 ([1], Theorem II.12). Let $L$ be a modular lattice and $X=$ $\{x, y, z\} \subseteq L$. Then:

(i) $\llbracket x \wedge y \wedge z, x \vee y \vee z \rrbracket_{X}$ is distributive if and only if $(x, y, z) D$,

(ii) $\llbracket x \wedge y \wedge z, x \vee y \vee z \rrbracket_{X}$ is distributive if and only if $(x, y, z) D^{*}$.

The Dedekind-Birkhoff Theorem shows that the hypothesis of modularity is necessary as well as sufficient in Theorem 1 (cf. the lattice (a) in Figure 1).

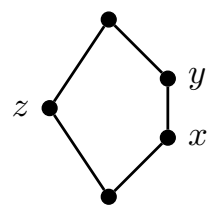

(a)

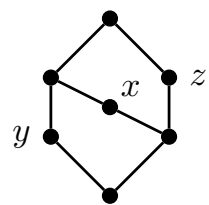

(b)

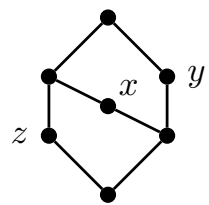

(c)

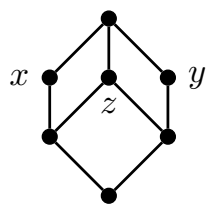

(d)

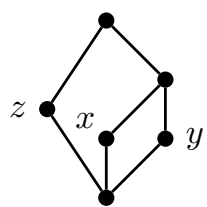

(e)

Fig. 1. Non-modular lattices satisfying $(x, y, z) D$ or $(x, y, z) D^{*}$.

Our result is the following.

Theorem 2. Let $L$ be an arbitrary lattice and $X=\{x, y, z\} \subseteq L$. Then:

(i) if $\llbracket x \wedge z, x \vee y \vee z \rrbracket_{X}$ and $\llbracket y \wedge z, x \vee y \vee z \rrbracket_{X}$ are distributive, then $(x, y, z) D$

(ii) if $\llbracket x \wedge y \wedge z, x \vee y \rrbracket_{X}$ is distributive, then $(x, y, z) D$.

ProOF: To prove (i), assume that $\llbracket x \wedge z, x \vee y \vee z \rrbracket_{X}$ and $\llbracket y \wedge z, x \vee y \vee z \rrbracket_{X}$ are distributive sublattices of $L$. Then

\footnotetext{
${ }^{1}$ Note that Birkhoff in [1], p. 37, provides a different definition: a three-element subset $\{x, y, z\}$ of a lattice $L$ is a distributive triple if $[\{x, y, z\}]$ is a distributive sublattice of $L$.
} 


$$
\begin{aligned}
z \wedge(x \vee y) & =z \wedge(x \vee(y \vee(x \wedge z))) \\
& =(z \wedge x) \vee(z \wedge(y \vee(x \wedge z))) \quad \text { (by the 1st assumption) } \\
& =z \wedge(y \vee(x \wedge z)) \\
& =z \wedge(y \vee((x \wedge z) \vee(y \wedge z))) \\
& =(z \wedge y) \vee(z \wedge((x \wedge z) \vee(y \wedge z)))
\end{aligned}
$$

(by the 2nd assumption)

$$
=(z \wedge y) \vee((x \wedge z) \vee(y \wedge z))
$$$$
=(z \wedge y) \vee(x \wedge z) \text {, }
$$

which completes the proof of (i).

For (ii), we assume that $\llbracket x \wedge y \wedge z, x \vee y \rrbracket_{X}$ is distributive and calculate as follows:

$$
\begin{aligned}
z \wedge(x \vee y) & =(z \wedge(x \vee y)) \wedge(x \vee y) \\
& =((z \wedge(x \vee y)) \wedge x) \vee((z \wedge(x \vee y)) \wedge y) \\
& =(z \wedge x) \vee(z \wedge y) .
\end{aligned}
$$

By the duality principle we obtain Theorem 3. Let $L$ be an arbitrary lattice and $X=\{x, y, z\} \subseteq L$. Then:

(i) if $\llbracket x \wedge y \wedge z, x \vee z \rrbracket_{X}$ and $\llbracket x \wedge y \wedge z, y \vee z \rrbracket_{X}$ are distributive, then $(x, y, z) D^{*}$

(ii) if $\llbracket x \wedge y, x \vee y \vee z \rrbracket_{X}$ is distributive, then $(x, y, z) D^{*}$.

REMARK 1. Lattices (b) and (c) in Figure 1 disprove the converses of Theorems 2 and 3, respectively.

REMARK 2. Theorem 2 allows the conclusion that $(x, y, z) D$ in lattices (d) and (e) in Figure 1. On the other hand, this fact cannot be justified on the basis of Theorem 1 .

In order to illustrate a possible use of Theorem 2 we will provide an easy inductive proof of the following 
TheOrem 4. Let $L$ be a lattice of finite length. If $L$ is modular but nondistributive lattice, then $L$ contains a covering diamond, i.e., a diamond $D=\{o, a, b, c, i\}$, such that $o \prec a, b, c \prec i$.

In the literature of lattice theory the preceding theorem is known as "folklore" (cf. [4], p. 111, or [2], p. 270). This theorem easily follows from [5] (cf. Theorem 1.4 for the case $n=2$ ), or from [3] (cf. Lemma 8, p. 247). Note that [6] generalizes the theorem to the class of weakly atomic lattices.

Proof of Theorem 4: Induction on $l(L)$ - the length of $L$. If $l(L)=1$ or $l(L)=2$ the theorem is obvious. For the induction step, assume that for any modular, non-distributive lattice $K$ if $l(K)<n$, then $K$ contains a covering diamond. Moreover, fix a modular, non-distributive lattice $L$ such that $l(L)=n \geqslant 3$. Then, by Dedekind-Birkhoff Theorem, $L$ contains a diamond $D=\{o, a, b, c, i\}$. If $0<o$ or $i<1$, then $[o, i]$ satisfies premises of our induction hypothesis, thus it contains a covering diamond, so $L$ does. If not, i.e., $D=\{0, a, b, c, 1\}$, since $l(L) \geqslant 3$ there exists some intermediate element $x \notin D$; we may assume without loss of generality that $b<x<1$.

Let us observe that $a \wedge x>0$, because if not, the set $\{0, a, x, b, 1\}$ would be a pentagon. For similar reasons, $c \wedge x>0$. Now, consider intervals $[a \wedge x, 1]$ and $[c \wedge x, 1]$. If one of them is non-distributive, then by the induction hypothesis, it contains a covering diamond, so $L$ does. On the other hand, if both intervals are distributive, then by Theorem 2 , the triple $(a, c, x)$ is distributive, thus we obtain

$$
(a \wedge x) \vee(c \wedge x)=(a \vee c) \wedge x=1 \wedge x=x .
$$

Moreover, by modularity, we get $(a \wedge x) \vee b=x$ and $(c \wedge x) \vee b=x$, and obviously $(a \wedge x) \wedge(c \wedge x)=(a \wedge x) \wedge b=(c \wedge x) \wedge b=0$, so the set $\{0, a \wedge x, b, c \wedge x, x\}$ forms a diamond. Therefore, by the induction hypothesis, the interval $[0, x]$ contains a covering diamond, and hence $L$ does.

Acknowledgements. I am grateful to the anonymous referees for their remarks, in particular, for drawing my attention to the paper [5], and the significant simplification of the proof of Theorem 2 . 


\section{References}

[1] G. Birkhoff, Lattice Theory, American Mathematical Society Colloquium Publications, Vol. XXV, Providence, Rhode Island (1973).

[2] E. Fried, G. Grätzer, H. Lakser, Projective geometries as cover-preserving sublattices, Algebra Universalis, Vol. 27 (1990), pp. 270-278.

[3] G. Grätzer, General lattice theory, Birkhäuser, Basel, Stuttgart (1978).

[4] G. Grätzer, Lattice Theory: Foundation, Birkhäuser, Basel (2011).

[5] C. Herrmann, A. P. Huhn, Lattices of normal subgroups which are generated by frames, [in:] Proceedings of the Lattice Theory Colloquium, Szeged 1974, Colloq. Math. Soc. János Bolyai, 14, pp. 97-136, NorthHolland, Amsterdam (1976).

[6] B. Jónsson, Equational Classes of Lattices, Mathematica Scandinavica, Vol. 22 (1968), pp. 187-196.

[7] M. Stern, Semimodular Lattices. Theory and Applications, Cambridge University Press (1999).

Department of Logic and Methodology of Sciences

University of Wrocław, Poland

e-mail: lazarzmarcin@poczta.onet.pl 